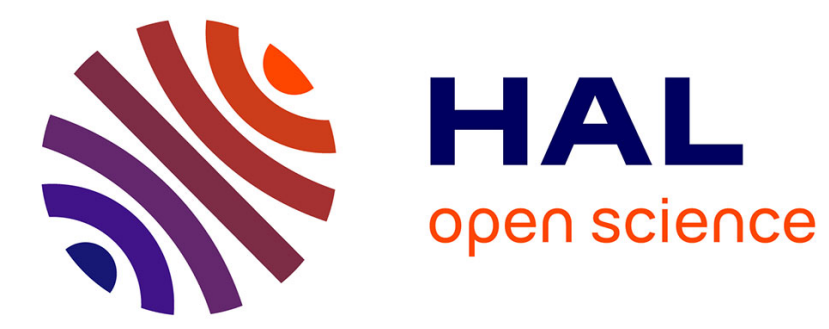

\title{
Reception filter impact on widely linear FRESH receiver performance for SAIC/MAIC with frequency offsets
}

\author{
Pascal Chevalier, Jean-Pierre Delmas, Rémi Chauvat
}

\section{To cite this version:}

Pascal Chevalier, Jean-Pierre Delmas, Rémi Chauvat. Reception filter impact on widely linear FRESH receiver performance for SAIC/MAIC with frequency offsets. SAM 2016: 9th IEEE Sensor Array and Multichannel Signal Processing Workshop, Jul 2016, Rio De Janeiro, Brazil. pp.1 - 5, 10.1109/SAM.2016.7569723 . hal-01391260

\section{HAL Id: hal-01391260 \\ https://hal.science/hal-01391260}

Submitted on 3 Nov 2016

HAL is a multi-disciplinary open access archive for the deposit and dissemination of scientific research documents, whether they are published or not. The documents may come from teaching and research institutions in France or abroad, or from public or private research centers.
L'archive ouverte pluridisciplinaire HAL, est destinée au dépôt et à la diffusion de documents scientifiques de niveau recherche, publiés ou non, émanant des établissements d'enseignement et de recherche français ou étrangers, des laboratoires publics ou privés. 


\title{
RECEPTION FILTER IMPACT ON WIDELY LINEAR FRESH RECEIVER PERFORMANCE FOR SAIC/MAIC WITH FREQUENCY OFFSETS
}

\author{
Pascal Chevalier ${ }^{(1,2)}$, Jean-Pierre Delmas ${ }^{(3)}$ and Rémi Chauvat ${ }^{(1,2)}$ \\ (1) CNAM, CEDRIC laboratory, 75003 Paris, France \\ (2) Thales-Communications-Security, HTE/AMS/TCP, 92622 Gennevilliers, France \\ (3) Telecom SudParis, UMR CNRS 5157, 91011 Evry, France
}

\begin{abstract}
Widely linear (WL) receivers are able to fulfill single antenna interference cancellation (SAIC) of one rectilinear (R) (ASK, BPSK) or quasi-rectilinear (QR) (MSK, GMSK, OQAM) co-channel interference (CCI). In the presence of residual frequency offsets (FO), standard SAIC/MAIC receivers lose their efficiency and have to be extended using WL frequency shifted (FRESH) filtering, which has been done recently. However, in practice the observations are low-pass filtered before sampling and processing, which may degrade the performance. In this context, the purpose of the paper is twofold. The first one is to extend the previous pseudo MLSE-based WL FRESH receiver, for sources with differential FO, to observations which are low-pass filtered. The second one is to analyze, both analytically and by simulations, the impact of the low-pass filtering on the performance of the extended pseudo MLSE-based WL FRESH receiver.
\end{abstract}

Index Terms - Widely linear, SAIC/MAIC, Frequency offset, Rectilinear, CCI, Pseudo-MLSE, FRESH, Low-Pass Filter

\section{INTRODUCTION}

These two last decades, since the pioneer works on the subject [1-4], WL filtering has aroused a great interest for second-order (SO) non-circular signals [5] in many areas. In particular, WL filters have been used efficiently for CCI mitigation in radio communication networks using $\mathrm{R}$ or $\mathrm{QR}$ modulations. Let us recall that $\mathrm{R}$ modulations correspond to mono-dimensional modulations such as ASK or BPSK modulations, whereas QR modulations are complex modulations corresponding, after a simple derotation operation, to a complex filtering of an $\mathrm{R}$ modulation. Examples of $\mathrm{QR}$ modulations are MSK, GMSK or OQAM modulations. WL filtering is able to fulfill SAIC of one R or QR multi-user CCI, allowing the separation of two users from only one receive antenna [6-8]. The effectiveness of this technology jointly with its low complexity are the reasons why it is operational in most of GSM handsets, generating significant networks capacity gains for the GSM system [8], [9]. Extension of the SAIC technology to a multi-antenna reception is called multiple antenna interference cancellation (MAIC). However, it has been shown recently in [10], in the GSM context, that in the presence of a differential frequency offset between the signal of interest (SOI) and the CCI, standard SAIC/MAIC receivers may lose their efficiency. This may be the case for airborne communications, due to high differential Doppler shifts between the sources. This is also the case in the context of filter bank multi-carrier (FBMC) waveforms, coupled with OQAM modulation, which are considered as promising candidates for the 5G mobile networks in particular [11]. Indeed, the Inter-Carrier Interference of FBMC-OQAM waveforms, which are difficult to remove for highly frequency selective channels or for MIMO systems, have FO corresponding to multiple of $50 \%$ of the (real) baud rate. In the presence of a differential FO between the sources, the SAIC/MAIC receivers have to be extended using WL FRESH receivers. Such a WL FRESH receiver has been introduced recently in [12], for R and QR signals and for arbitrary propagation channels, using the pseudo-MLSE approach introduced in [13-14]. Performance of this receiver, jointly with the impact of the FO on the latter, have been analyzed both analytically and by simulations, showing off the great interest of this receiver whatever the value of the FO. However in practice, the observations are low-pass filtered before sampling and processing. One may then wonder whether this low-pass filtering operation may degrade or not the performance of the considered WL FRESH receiver.

In this context, the purpose of this paper is twofold. The first one is to extend, for R sources with different FO, the WL FRESH receiver introduced in [12] to observations which are low-pass filtered. The second one is to analyze, both analytically and by simulations, the impact of the low-pass filtering on the performance of the extended WL FRESH receiver. Note that papers dealing with WL FRESH filtering for equalization/demodulation in presence of CCI are not so numerous and correspond to [15-18] for R signals and [19-21] for QR signals. However none of these papers discuss the impact of a low-pass filter on the output performance.

\section{MODELS AND STATISTICS}

\subsection{Observation model and SO statistics}

We consider an array of $N$ narrow-band antennas receiving the contribution of a SOI, one CCI and a background noise. Both sources are assumed to be R. The vector of complex 
amplitudes of the data at the output of these antennas after frequency synchronization can then be written as

$$
\begin{aligned}
\mathbf{x}(t) & =\sum_{k}\left[b_{k} \mathbf{g}(t-k T)+e_{k}\left(v(t-k T) e^{j 2 \pi \Delta_{f} t}\right) * \mathbf{h}_{I}(t)\right]+\mathbf{u}(t) \\
& =\sum_{k} b_{k} \mathbf{g}(t-k T)+\sum_{k} e_{k} e^{j 2 \pi \Delta_{f} k T} \mathbf{g}_{I_{o}}(t-k T)+\mathbf{u}(t) \\
& \triangleq \sum_{k} b_{k} \mathbf{g}(t-k T)+\mathbf{n}(t) .
\end{aligned}
$$

Here, $b_{k}$ and $e_{k}$ are real-valued zero-mean i.i.d. r.v., corresponding to the SOI and CCI symbols respectively, $T$ is the symbol period, $\mathbf{g}(t)=v(t) * \mathbf{h}(t)$ is the impulse response of the SOI global channel, $*$ is the convolution operation, $v(t)$ and $\mathbf{h}(t)$ are the impulse responses of the SOI pulse shaping filter and propagation channel respectively, $\Delta_{f}$ is the residual FO of the CCI, which is assumed to be known and positive or null, $\mathbf{g}_{I_{o}}(t)=v_{o}(t) * \mathbf{h}_{I}(t)$ where $v_{o}(t)=v(t) e^{j 2 \pi \Delta_{f} t}$ and $\mathbf{h}_{I}(t)$ is the impulse response of the propagation channel of the CCI, $\mathbf{u}(t)$ is the background noise vector, assumed zeromean, circular, stationary, temporally and spatially white and $\mathbf{n}(t)$ is the total noise vector composed of the CCI and background noise.

The observations are assumed to be filtered by an ideal low-pass filter having a bandwidth $B^{\prime}$ whose impulse response is $p(t)$ and whose frequency response is $p(f)$, as depicted by Figure 1. Denoting by $B$ the bandwidth of the SOI, the response $p(f)$ is equal to 1 for $-B^{\prime} / 2 \leq f \leq B^{\prime} / 2$ and to 0 elsewhere, where $B \leq B^{\prime}$. The low-pass filtered observation vector is then given by

$$
\mathbf{x}_{p}(t) \triangleq \mathbf{x}(t) * p(t)=\sum_{k} b_{k} \mathbf{g}(t-k T)+\mathbf{n}_{p}(t)
$$

where $\mathbf{n}_{p}(t) \triangleq \mathbf{n}(t) * p(t)$.

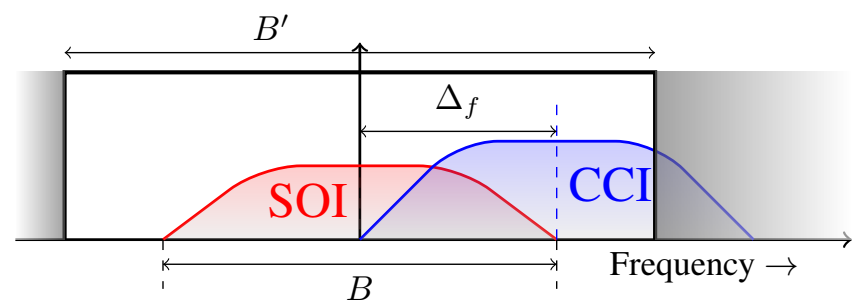

Fig. 1. Spectral representation of the SOI, CCI and $p(t)$

The SO statistics of $\mathbf{n}_{p}(t)$ are characterized by the two correlation matrices $\mathbf{R}_{n}(t, \tau)$ and $\mathbf{C}_{n}(t, \tau)$, defined by

$$
\begin{aligned}
& \mathbf{R}_{n}(t, \tau) \triangleq \mathrm{E}\left[\mathbf{n}_{p}(t+\tau / 2) \mathbf{n}_{p}^{H}(t-\tau / 2)\right] \\
& \mathbf{C}_{n}(t, \tau) \triangleq \mathrm{E}\left[\mathbf{n}_{p}(t+\tau / 2) \mathbf{n}_{p}^{T}(t-\tau / 2)\right]
\end{aligned}
$$

where $(.)^{T}$ and $(.)^{H}$ mean transpose and conjugate transpose respectively. Using (1), it is easy to verify that $\mathbf{R}_{n}(t, \tau)$ is a periodic function of $t$ with a period equal to $T$. In a same way, it is easy to show that $\mathbf{C}_{n}(t, \tau)=\mathbf{C}_{n}^{\prime}(t, \tau) e^{j 4 \pi \Delta_{f} t}$ where $\mathbf{C}_{n}^{\prime}(t, \tau)$ is a periodic function of $t$ with a period equal to $T$. Matrices $\mathbf{R}_{n}(t, \tau)$ and $\mathbf{C}_{n}(t, \tau)$ have then Fourier series expansions given by

$$
\begin{aligned}
& \mathbf{R}_{n}(t, \tau)=\sum_{\alpha_{i}} \mathbf{R}_{n}^{\alpha_{i}}(\tau) e^{j 2 \pi \alpha_{i} t} \\
& \mathbf{C}_{n}(t, \tau)=\sum_{\beta_{i}} \mathbf{C}_{n}^{\beta_{i}}(\tau) e^{j 2 \pi \beta_{i} t} .
\end{aligned}
$$

Here, $\alpha_{i}$ and $\beta_{i}$ are the SO and conjugate SO cyclic frequencies of $\mathbf{n}_{p}(t)$ such that $\alpha_{i}=i / T$ and $\beta_{i}=i / T+2 \Delta_{f}(i \in \mathbb{Z})$, $\mathbf{R}_{n}^{\alpha_{i}}(\tau)$ and $\mathbf{C}_{n}^{\beta_{i}}(\tau)$ are respectively the cyclic and conjugate cyclic correlation matrices of $\mathbf{n}_{p}(t)$ for the cyclic frequencies $\alpha_{i}$ and $\beta_{i}$ and the delay $\tau$, defined by

$$
\begin{aligned}
& \mathbf{R}_{n}^{\alpha_{i}}(\tau) \triangleq\left\langle\mathbf{R}_{n}(t, \tau) e^{-j 2 \pi \alpha_{i} t}\right\rangle \\
& \mathbf{C}_{n}^{\beta_{i}}(\tau) \triangleq\left\langle\mathbf{C}_{n}(t, \tau) e^{-j 2 \pi \beta_{i} t}\right\rangle
\end{aligned}
$$

where $\langle\cdot\rangle$ is the temporal mean operation in $t$ over an infinite observation duration.

\subsection{Conventional and two-input FRESH models}

Conventional linear processing of $\mathbf{x}_{p}(t)$ only exploits the information contained in the zero $(\alpha=0)$ cyclic frequency of $\mathbf{x}_{p}(t)$.

The WL FRESH receiver introduced in [12] for R signals exploits the conjugate SO cyclic frequency $\beta=2 \Delta_{f}$ of $\mathbf{x}_{p}(t)$ through the exploitation of the two-input FRESH observation vector $\mathbf{x}_{p, F_{2}}(t)$, defined by

$$
\begin{aligned}
\mathbf{x}_{p, F_{2}}(t) & \triangleq\left[\mathbf{x}_{p}^{T}(t), e^{j 4 \pi \Delta_{f} t} \mathbf{x}_{p}^{H}(t)\right]^{T} \\
& =\sum_{k} b_{k} \mathbf{g}_{F_{2}, k}(t-k T)+\mathbf{n}_{p, F_{2}}(t)
\end{aligned}
$$

Here, $\mathbf{n}_{p, F_{2}}(t) \triangleq\left[\mathbf{n}_{p}^{T}(t), e^{j 4 \pi \Delta_{f} t} \mathbf{n}_{p}^{H}(t)\right]^{T}, \mathbf{g}_{F_{2}, k}(t) \triangleq$ $\left[\mathbf{g}^{T}(t), e^{j 4 \pi \Delta_{f}(t+k T)} \mathbf{g}^{H}(t)\right]^{T}$.

\section{GENERIC PSEUDO-MLSE RECEIVER}

\subsection{Generic pseudo-MLSE receiver}

We denote by $\mathbf{x}_{p, F_{M}}(t)$ and $\mathbf{n}_{p, F_{M}}(t)$ the generic $M(M=$ $1,2)$ input FRESH observation and associated FRESH total noise vectors respectively. We assume that $\mathbf{x}_{p, F_{1}}(t)$ and $\mathbf{n}_{p, F_{1}}(t)$ correspond to $\mathbf{x}_{p}(t)$ and $\mathbf{n}_{p}(t)$ respectively. Thus for $M=1$, we consider conventional linear receivers whereas for $M=2$ we consider two-input WL FRESH receivers. We denote by $\mathbf{R}_{F_{M}}^{0}(f)$ the Fourier transform of (7), where $\alpha_{i}$ and $\mathbf{n}(t)$ are replaced by 0 and $\mathbf{n}_{p, F_{M}}(t)$ respectively.

To apply the standard pseudo-MLSE criterion introduced in [13], we have to elaborate a bit. Indeed, it is straigthforward to verify that, depending on $\Delta_{f}$ and $B^{\prime}$, matrix $\mathbf{R}_{F_{2}}^{0}(f)$ may not be always invertible for $-B / 2 \leq f \leq B / 2$ and $2 \Delta_{f}-$ $B / 2 \leq f \leq 2 \Delta_{f}+B / 2$. This prevents to easily compute the pseudo-MLSE two-input FRESH receiver from (2). To solve this problem, we consider the following artificial two-input FRESH observation vector 


$$
\begin{aligned}
\mathbf{x}_{p, F_{M}, \delta}(t) & \triangleq \mathbf{x}_{p, F_{M}}(t)+\mathbf{n}_{F_{M}, \delta}(t) \\
& =\sum_{k} b_{k} \mathbf{g}_{F_{M}, k}(t-k T)+\mathbf{n}_{p, F_{M}, \delta}(t)
\end{aligned}
$$

where, for $1 \leq M \leq 2, \mathbf{n}_{F_{M}, \delta}(t)$ is a $(M N \times 1)$ artificial noise vector, assumed to be Gaussian, circular, stationary, spatially and temporally white with a power spectral density equal to $N_{\delta}$, whereas $\mathbf{n}_{p, F_{M}, \delta}(t)=\mathbf{n}_{p, F_{M}}(t)+\mathbf{n}_{F_{M}, \delta}(t)$. Vector $\mathbf{x}_{p, F_{M}}(t)$ becomes the limit of $\mathbf{x}_{p, F_{M}, \delta}(t)$ as $N_{\delta} \rightarrow 0$. Assuming a stationary, circular and Gaussian generic FRESH total noise $\mathbf{n}_{p, F_{M}}(t)$, vector $\mathbf{n}_{p, F_{M}, \delta}(t)$ has the same properties. It is shown in [22], [23] that the sequence $\mathbf{b} \triangleq$ $\left(b_{1}, \ldots, b_{K}\right)$ which maximizes its likelihood from $\mathbf{x}_{p, F_{M}, \delta}(t)$ is the one which minimizes the following criterion ${ }^{1}$ :

$$
\begin{aligned}
C_{\delta}(\mathbf{b})=\int\left[\mathbf{x}_{p, F_{M}, \delta}(f)\right. & \left.-\mathbf{s}_{F_{M}}(f)\right]^{H}\left[\mathbf{R}_{F_{M}, \delta}^{0}(f)\right]^{-1} \\
& \times\left[\mathbf{x}_{p, F_{M}, \delta}(f)-\mathbf{s}_{F_{M}}(f)\right] d f .
\end{aligned}
$$

Here, $\mathbf{R}_{F_{M}, \delta}^{0}(f)$ corresponds to the Fourier transform of (7), where $\alpha_{i}$ and $\mathbf{n}(t)$ are replaced by 0 and $\mathbf{n}_{p, F_{M}, \delta}(t)$ respectively. This matrix is always invertible for $N_{\delta} \neq 0$ whatever the value of $f$, while $\mathbf{s}_{F_{M}}(f) \triangleq \sum_{k=1}^{K} b_{k} \mathbf{g}_{F_{M}, k}(f) e^{-j 2 \pi f k T}$, where $\mathbf{g}_{F_{M}, k}(f)$ corresponds to $\mathbf{g}(f)$ for $M=1$. A sufficient statistics of (11) is obtained by integrating (11) on $[-B / 2, B / 2]$ and $\left[2 \Delta_{f}-B / 2,2 \Delta_{f}+B / 2\right]$ only. For subbands for which $p(f)$ and $p\left(2 \Delta_{f}-f\right)$ overlap inside $[-B / 2, B / 2]$ or $\left[2 \Delta_{f}-B / 2,2 \Delta_{f}+B / 2\right], \mathbf{R}_{F_{M}}^{0}(f)$ is always invertible whatever $M$ and the limit of $C_{\delta}(\mathbf{b})$ when $N_{\delta} \rightarrow 0$ can be taken directly for these subbands. However, for subbands for which $p(f)$ and $p\left(2 \Delta_{f}-f\right)$ do not overlap inside $[-B / 2, B / 2]$ or $\left[2 \Delta_{f}-B / 2,2 \Delta_{f}+B / 2\right] \mathbf{R}_{F_{2}}^{0}(f)$ is not invertible. For these subbands, it is then necessary to take a sufficient statistics of (11) keeping only terms that depend on the symbols $b_{k}$, before taking the limit of the obtained sufficient statistics when $N_{\delta} \rightarrow 0$. Considering, for each subband, only terms that depend on the symbols $b_{k}$, it is possible to show, after some manipulations, that the minimization of a sufficient statistics of (11) when $N_{\delta} \rightarrow 0$ is equivalent to that of the metric:

$$
\Lambda(\mathbf{b})=\sum_{k=1}^{K} \sum_{k^{\prime}=1}^{K} b_{k} b_{k^{\prime}} r_{k, k^{\prime}}-2 \sum_{k=1}^{K} b_{k} z_{p, F_{M}}(k)
$$

where $z_{p, F_{M}}(k) \triangleq \operatorname{Re}\left[y_{p, F_{M}}(k)\right]$ and where the sampled output $y_{p, F_{M}}(k)$ and $r_{k, k^{\prime}}$ are defined by

$$
\begin{gathered}
y_{p, F_{M}}(k)=\int \mathbf{w}_{F_{M}, k}^{H}(f) \mathbf{x}_{p, F_{M}}(f) e^{j 2 \pi f k T} d f \\
r_{k, k^{\prime}}=\int \mathbf{w}_{F_{M}, k}^{H}(f) \mathbf{g}_{F_{M}, k^{\prime}}(f) e^{j 2 \pi f\left(k-k^{\prime}\right) T} d f .
\end{gathered}
$$

The vectors $\mathbf{w}_{F_{M}, k}(f), 1 \leq M \leq 2$, are defined by

$$
\mathbf{w}_{F_{1}, k}(f) \triangleq \mathbf{R}_{F_{1}}^{0}(f)^{-1} \mathbf{g}(f) \triangleq \mathbf{w}_{F_{1}}(f)
$$

\footnotetext{
${ }^{1}$ All Fourier transforms of vectors $\mathbf{x}$ and matrices $\mathbf{X}$ use the same notation where $t$ or $\tau$ is simply replaced by $f$.
}

$$
\begin{aligned}
& \text { If } B^{\prime} \leq 4 \Delta_{f}-B \\
& \mathbf{w}_{F_{2}, k}(f) \triangleq\left[\mathbf{w}_{F_{1}}^{T}(f), \mathbf{0}^{T}\right]^{T} ; \quad-B / 2 \leq f \leq B / 2 \\
& \mathbf{w}_{F_{2}, k}(f) \triangleq\left[\mathbf{0}^{T}, \mathbf{w}_{F_{1}}^{H}\left(2 \Delta_{f}-f\right) e^{j 4 \pi \Delta_{f} k T}\right]^{T} ; \\
& \quad 2 \Delta_{f}-B / 2 \leq f \leq 2 \Delta_{f}+B / 2
\end{aligned}
$$

\subsection{Interpretation of the generic pseudo-MLSE receiver}

We deduce from (13) that $y_{p, F_{M}}(k)$ is the sampled version, at time $t=k T$, of the output of the filter whose frequency response is $\mathbf{w}_{F_{M}, k}^{H}(f)$, where $\mathbf{w}_{F_{M}, k}(f)$ is defined by (15) to (21) and whose input is $\mathbf{x}_{p, F_{M}}(t)$. It is composed of the WL FRESH filter $\mathbf{w}_{F_{M}, k}^{H}(f)$, which reduces to a time-invariant (TI) linear filter for conventional receivers, followed by a sampling at the symbol rate, a real part capture and a decision box implementing the Viterbi algorithm, since $r_{k, k^{\prime}}^{*}=r_{k^{\prime}, k}$.

\subsection{SINR at the output of the generic pseudo-MLSE re- ceiver}

For real-valued symbols $b_{k}$, the symbol error rate (SER) at the output of the generic $M$ input $(M=1,2)$ pseudo-MLSE receiver is directly linked to the signal to interference plus noise ratio (SINR) on the current symbol before decision, i.e. at the output $z_{p, F_{M}}(n)$ [24, Sec. 10.1.4], while the inter-symbol interference is processed by the decision box. For this reason, we compute the general expression of the output SINR hereafter and we analyse its variations in section IV. It is easy to verify from (1), (9), (13) and (14) that $z_{p, F_{M}}(n)$ can be written as

$$
z_{p, F_{M}}(n)=b_{n} r_{n, n}+\sum_{k \neq n} b_{k} \operatorname{Re}\left[r_{n, k}\right]+z_{n, p, F_{M}}(n)
$$

where the real-valued sample $z_{n, p, F_{M}}(n)$ is defined by the real part of (13) for $k=n$ with $\mathbf{n}_{p, F_{M}}(f)$ instead of $\mathbf{x}_{p, F_{M}}(f)$. Defining $\pi_{b} \triangleq \mathrm{E}\left[b_{n}^{2}\right]$, the SINR on the current symbol $n$ is then given by

$$
\operatorname{SINR}_{F_{M}, n} \triangleq \pi_{b} r_{n, n}^{2} / \mathrm{E}\left[z_{n, p, F_{M}}^{2}(n)\right] .
$$

\section{SINR ANALYSIS}

\subsection{Assumptions}

In this section, we analyse both analytically and by simulations, the impact of the parameter $B^{\prime}$ on the SINR at the 
output of the two-input pseudo-MLSE receiver. For this purpose, we consider the model (1) and we assume a raised cosine pulse shaping filter $v(t)$ with a roll-off $\gamma$. The SOI and CCI have the same bandwidth, $B=(1+\gamma) / T$, and spectrally overlap if $0 \leq \Delta_{f} \leq B$, (i.e. if $0 \leq \Delta_{f} T \leq 1+\gamma$ ) as illustrated in Fig. 1, what we assume in the following. Moreover, to easily describe the behavior and quantify the performance of the generic $M$ input pseudo-MLSE receiver, we limit the analysis to deterministic propagation channels with no delay spread such that

$$
\mathbf{h}(t)=\mu \delta(t) \mathbf{h} \text { and } \mathbf{h}_{I}(t)=\mu_{I} \delta\left(t-\tau_{I}\right) \mathbf{h}_{I} .
$$

Here, $\mu$ and $\mu_{I}$ control the amplitude of the SOI and CCI, $\delta(t)$ is the Dirac pulse, $\tau_{I}$ is the delay of the CCI with respect to the SOI whereas $\mathbf{h}$ and $\mathbf{h}_{I}$, such that $\mathbf{h}^{H} \mathbf{h}=\mathbf{h}_{I}^{H} \mathbf{h}_{I}=N$, are the channel vectors of the SOI and CCI.

\subsection{SINR computations and analysis for a zero roll-off}

Under the previous assumptions, analytical interpretable expressions of the $\operatorname{SINR}_{F_{M}, n}$ (23) are only possible for a zero roll-off $\gamma$ which is assumed in this subsection. For a zero roll-off, for which $B=1 / T$, the quantities $\pi_{s} \triangleq \mu^{2} \pi_{b}$, $\pi_{I} \triangleq \mu_{I}^{2} \pi_{e}$ and $\eta_{2}$ correspond to the mean power of the SOI, the CCI (for $\Delta_{f}=0$ ) and the background noise per antenna at the output of the pulse shaping matched filter respectively, where $\pi_{e} \triangleq \mathrm{E}\left[e_{n}^{2}\right]$. We then denote by $\phi_{s I}$, the phase of $\mathbf{h}^{H} \mathbf{h}_{I}, \varepsilon_{s}$ and $\varepsilon_{I}$ the quantities $\varepsilon_{s} \triangleq \pi_{s} \mathbf{h}^{H} \mathbf{h} / \eta_{2}$ and $\varepsilon_{I} \triangleq \pi_{I} \mathbf{h}_{I}^{H} \mathbf{h}_{I} / \eta_{2}$ and by $\operatorname{SINR}_{R_{M}, n}$ the SINR (23) at the output of the $M$ input pseudo-MLSE receiver at time $n T$. Due to lack of space, we limit the analysis to $N=1$ antenna. Assuming a strong CCI $\left(\varepsilon_{I} \gg 1\right)$, we obtain, after tedious computations not reported here

$$
\begin{aligned}
& \mathrm{SINR}_{1, n} \approx 2 \varepsilon_{s} \Delta_{f} T ; \quad \Delta_{f} \neq 0 \\
& \operatorname{SINR}_{1, n}=2 \varepsilon_{s} /\left[1+2 \varepsilon_{I} \cos ^{2}\left(\phi_{s I}\right)\right] ; \quad \Delta_{f}=0 \\
& \mathrm{SINR}_{2, n} \approx 2 \varepsilon_{s}\left[1-\frac{\frac{3-T B^{\prime}}{2}+\left(1-2 \Delta_{f} T\right) \cos \left(2 \Psi_{s I, n}\right)}{2}\right] \text {; } \\
& B \leq B^{\prime} \leq 2 \Delta_{f}+B \\
& \mathrm{SINR}_{2, n} \approx 2 \varepsilon_{s}\left[1-\frac{1-\Delta_{f} T+\left(1-2 \Delta_{f} T\right) \cos \left(2 \Psi_{s I, n}\right)}{2}\right] \text {; } \\
& 2 \Delta_{f}+B \leq B^{\prime} \\
& \mathrm{SINR}_{2, n} \approx 2 \varepsilon_{s} \Delta_{f} T ; \quad \Delta_{f} \neq 0 ; B \leq B^{\prime} \leq 4 \Delta_{f}-B \\
& \mathrm{SINR}_{2, n} \approx 2 \varepsilon_{s}\left[1-\left(3-T B^{\prime}\right) / 4\right] ; 4 \Delta_{f}-B \leq B^{\prime} \leq 2 \Delta_{f}+B \\
& \mathrm{SINR}_{2, n} \approx 2 \varepsilon_{s}\left[1-\left(1-\Delta_{f} T\right) / 2\right] ; \quad 2 \Delta_{f}+B \leq B^{\prime}
\end{aligned}
$$

where $\Psi_{s I, n}$ is defined by

$$
\Psi_{s I, n} \triangleq \phi_{s I}+2 \pi \Delta_{f}\left(n T-\tau_{I}\right)
$$

Let us recall that a receiver completely cancels the CCI as $\varepsilon_{I} \rightarrow \infty$, or performs SAIC, at time $n T$ if the associated
$\mathrm{SINR}_{n}$ does not converge toward zero. Previous expressions show that $\mathrm{SINR}_{2, n}$ is always an increasing function of $B^{\prime}$ corresponding to the SINR without any filtering for $B^{\prime} \geq$ $2 \Delta_{f}+B$, i.e. as soon as the low-pass filter does not cut the CCI spectrum. In this case the spectral correlation properties of the CCI are not altered by the low-pass filter. However, for $B^{\prime}<2 \Delta_{f}+B$, the CCI spectrum is cut by the lowpass filter, which modifies the spectral correlation properties of the CCI and which deteriorates the output SINR of the WL FRESH receiver. In this case, the performance degradation increases as $B^{\prime}$ decreases toward $B$. Moreover, expression (29) shows that for $B / 2 \leq \Delta_{f} \leq B$, SINR $_{2, n}$ corresponds to the SINR at the output of the conventional receiver as soon as $B^{\prime} \leq 4 \Delta_{f}-B$. In this case the low-pass filtering operation has completely removed the spectral correlation properties of the CCI inside the SOI bandwidth and then the interest of the WL FRESH receiver. Figure 2 illustrates these results by showing the variations of $\operatorname{SINR}_{2, n}$ as a function of $B^{\prime}$ for $\phi_{s I}=\pi / 4, \tau_{I}=0$ and for several values of $\Delta_{f}$. Note the decreasing output SINR as $B^{\prime}$ decreases below $2 \Delta_{f}+B$. All these results show off the importance of a careful choice of the low-pass filter which has to keep the CCI unfiltered in order to maintain the whole potential of the WL FRESH receiver for SAIC.

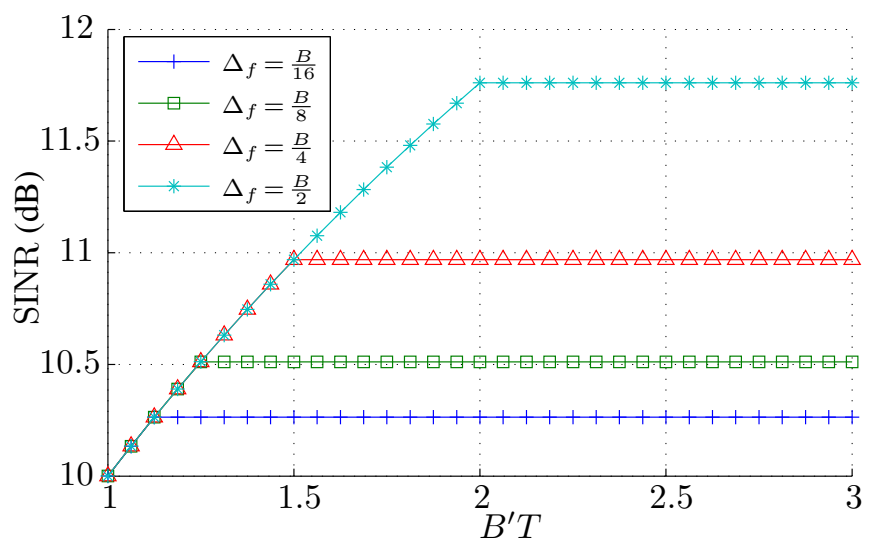

Fig. 2. $\mathrm{SINR}_{2, n}$ as a function of $B^{\prime} T, \varepsilon_{s}=10 \mathrm{~dB}, \varepsilon_{I}=20 \mathrm{~dB}$

\section{CONCLUSION}

The WL FRESH receiver introduced in [12] for SAIC/MAIC of a R CCI having a residual FO has been extended in this paper, for arbitrary propagation channels and from a pseudo MLSE-based approach, for low-pass filtered observations. Performance of the extended WL FRESH receiver have been analyzed for deterministic propagation channels with no delay spread, both analytically and by simulations, enlightening the impact of the low-pass filter bandwidth. It has been shown that to maintain the whole potential of the WL FRESH receiver introduced in [12], the low-pass filter has to keep the CCI unfiltered. Extension of these results for QR signals will be presented elsewhere. These results should be very helpful in particular to optimize the reception filter of FBMC-OQAM systems using WL filtering at reception. 


\section{REFERENCES}

[1] W.M. Brown and R.B. Crane, "Conjugate linear filtering”, IEEE Trans. Inf. Theory, vol. 15, no. 4, pp. 462-465, July. 1969.

[2] W.A. Gardner, "Cyclic Wiener filtering: theory and method", IEEE Trans. Commun., vol. 41, no. 1, pp. 151163, Jan. 1993.

[3] B. Picinbono and P. Chevalier, "Widely linear estimation with complex data", IEEE Trans. Signal Process., vol. 43, no. 8, pp. 2030-2033, Aug. 1995.

[4] P. Chevalier, "Optimal array processing for non stationary signals", Proc. ICASSP, pp. 2868-2871, Atlanta (USA), May 1996.

[5] B. Picinbono, "On Circularity", IEEE Trans. Signal Process., vol. 42, no. 12, pp. 3473-3482, Dec 1994.

[6] H. Trigui and D.T.M. Slock, "Performance bounds for cochannel interference cancellation within the current GSM standard", Signal Processing, Elsevier, vol. 80, pp. 1335-1346, 2000.

[7] P. Chevalier and F. Pipon, "New Insights into optimal widely linear array receivers for the demodulation of BPSK, MSK and GMSK signals corrupted by noncircular interferences - Application to SAIC", IEEE Trans. Signal Process., vol. 54, no. 3, pp. 870-883, March 2006.

[8] R. Meyer, W.H. Gerstacker, R. Schober, and J.B. Huber, "A single antenna interference cancellation algorithm for increased GSM capacity", IEEE Trans. Wireless Commun., vol. 5, no. 7, pp. 1616-1621, July 2006.

[9] A. Mostafa, R. Kobylinski, I. Kostanic, and M. Austin, "Single Antenna Interference Cancellation (SAIC) for GSM networks", IEEE Proc. Vehicular Technology Conference (VTC04), vol 2, pp. 1089-1093, Oct. 2004.

[10] JP. Delmas, S. Sallem and P. Chevalier, "Sensitivity of SAIC and MAIC Concepts to Residual Frequency Offsets", Proc. European Signal Processing Conference (EUSIPCO'10), Alborg (Denmark), Aug. 2010.

[11] G. Wunder et al. "5GNOW: Non-orthogonal asynchronous waveforms for future mobile applications", IEEE Commun. Magazine, pp. 97-105, Feb. 2014.

[12] R. Chauvat, P. Chevalier and JP. Delmas, Widely Linear FRESH Receiver for SAIC/MAIC with Frequency Offsets, International Symposium on Wireless Communication Systems (ISWCS15), Brussels, Aug. 2015.

[13] P. Chevalier, R. Chauvat and J.P. Delmas, "Quasirectilinear (MSK, GMSK, OQAM) co-channel interference mitigation by three inputs widely linear FRESH filtering", Proc. International Conference of Acoustic Speech and Signal Processing, Brisbane (Australia), April 2015.

[14] R. Chauvat, P. Chevalier and J.P. Delmas, "How to make quasi-rectilinear signals (MSK, GMSK, OQAM) almost equivalent to rectilinear ones (BPSK, ASK) for widely linear filtering in the presence of CCI", ITG Workshop on Smart Antennas (WSA'15), Ilmenau, Germany, March 2015.

[15] W.A. Gardner and W.A. Brown, 'Frequency-Shift Filtering Theory for Adaptive Co-Channel Interference Removal", 23th Asilomar Conference on Signals, Systems and Computers, Vol. 2, pp. 562-567, Nov. 1989.

[16] H.E. Wong and J.A. Chambers, "Two-stage interference immune blind equalizer which exploits cyclostationary statistics", Electronic Letters, Vol. 32, no. 19, pp. 17631764, Sept. 1996.

[17] G. Gelli, L. Paura and A.M. Tulino, "Cyclostationaritybased filtering for narrow-band interference suppression in direct sequence spread-spectrum systems", IEEE Journal on Selected Areas in Communications, Vol. 6, no. 9, pp. 1747-1755, Dec. 1998.

[18] G. Latouche, D. Pirez and P. Vila, "MMSE cyclic equalization", Proc. MILCOM, pp. 150-154, Boston, Oct. 1998.

[19] W.A. Gardner and C.W. Reed, "Making the most out of spectral redundancy in GSM: Cheap CCI suppression", Proc. ASILOMAR Conf., pp. 883-889, PacificGrove, 2001.

[20] A. Mirbagheri, K.N. Plataniotis, and S. Pasupathy, ”An enhanced Widely Linear CDMA receiver with OQPSK modulation", IEEE Trans. Commun., vol. 54, no. 2, pp. 261-272, Feb. 2006.

[21] A.U.H. Sheikh and F. Hendessi, "FRESH-DFE: a new structure for interference cancellation", Wireless Personal Communications Journal, vol. 44, pp. 101-118, 2008.

[22] S. Sallem, J.P. Delmas, and P. Chevalier, "Optimal SIMO MLSE receivers for the detection of linear modulation corrupted by noncircular interference," IEEE workshop on Statistical Signal Processing (SSP'2012), Ann Arbor, USA, Aug. 2012.

[23] G. Ungerboeck, "Adaptive maximum likelihood receiver for carrier-modulated data transmission systems", IEEE Trans. Commun., vol. 22, no. 5, pp. 624-636, May 1974.

[24] J.G. Proakis, Digital Communications, McGraw Hill Series in Electrical and Computer Engineering, 4th Edition, 2001. 